\title{
Benefits of on-wafer calibration standards fabricated in membrane technology
}

\author{
M. Rohland ${ }^{1,2}$, U. Arz ${ }^{1}$, and S. Büttgenbach ${ }^{2}$ \\ ${ }^{1}$ Physikalisch-Technische Bundesanstalt, 38116 Braunschweig, Germany \\ ${ }^{2}$ Institut für Mikrotechnik, Langer Kamp 8, 38106 Braunschweig, Germany
}

\begin{abstract}
In this work we compare on-wafer calibration standards fabricated in membrane technology with standards built in conventional thin-film technology. We perform this comparison by investigating the propagation of uncertainties in the geometry and material properties to the broadband electrical properties of the standards. For coplanar waveguides used as line standards the analysis based on Monte Carlo simulations demonstrates an up to tenfold reduction in uncertainty depending on the electromagnetic waveguide property we look at.
\end{abstract}

\section{Introduction}

Traceability for scattering parameter measurements back to SI units has long been possible for coaxial waveguides. The characteristic impedance can be calculated very accurately from the cross-sectional dimensions of the coaxial geometry. It is in principle also possible to determine the properties of coplanar waveguides to a high degree of accuracy.

However, uncertainties in the complex permittivity of the dielectric substrate can have a substantial effect on the propagation characteristics of the coplanar waveguide (CPW) as recent studys (Arz et al., 2008a) have shown. Even when using state-of-the-art measurement techniques (Arz et al., 2008b) to accurately capture the frequency-dependent permittivity and loss tangent of the substrate, the remaining uncertainties in the waveguide properties are still much higher than in the coaxial airline case.

A carrier substrate beneath the coplanar lines with the effective dielectric constant value close to the ideal value of 1 provides one possible solution of this problem. Such a carrier substrate would lead to excellent dispersion properties and

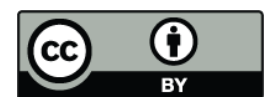

Correspondence to: M.Rohland (m.rohland@tu-bs.de) also prevent the excitation of undesired surface modes. A good approximation of these ideal structures are CPWs fabricated on thin insulating carrier membranes. These coplanar waveguides have been built successfully. The applied technologies are normally used for fabricating microelectromechanical systems (MEMS).

\section{CPW fabrication}

The technology for fabricating membrane-supported CPWs is currently being developed in a collaboration with the Institute for Microtechnology in Braunschweig, Germany. The cross section of a coplanar airline built in membrane technology together with its geometrical and material parameters is shown in Fig. 1. The CPW is supported by a thin film stretched across a silicon frame. In order to meet the requirements, the layer must possess good electrical and mechanical properties. The thin film must have low losses at microwave and millimeter-wave frequencies in order to achieve superior electrical performance, as well as be compatible with semiconducting and conducting materials. Reduced sensitivity to applied pressure and temperature variations, along with increased membrane sizes must be considered for optimization of the mechanical properties. Therefore, we use a thin film of silicon nitride produced by plasma enhanced chemical vapor deposition (PECVD) at multi-frequency (mf-nitride), made available by Rohde $\&$ Schwarz, to process membrane supported CPWs.

In contrary to the well-known three-layer approaches described in Dib et al. (1991); Weller et al. (1994); Katehi and Rebeiz (1996), our approach consists of one layer. The threelayer membranes in Dib et al. (1991); Weller et al. (1994); Katehi and Rebeiz (1996) use three process steps to deposit one layer of thermal oxide, one layer of low pressure chemical vapor deposition (LPCVD) silicon nitride and one layer of LPCVD silicon oxide. 


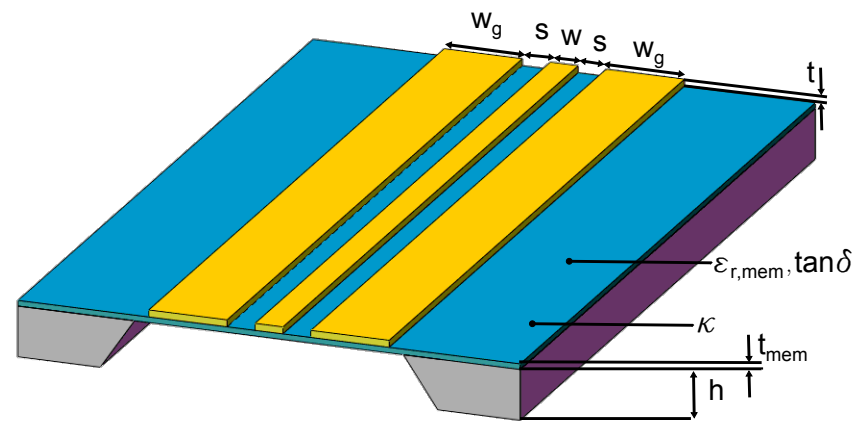

Fig. 1. Cross section of CPW based on membrane technology.

The mf-nitride used in this work is one dielectric layer which is deposited in one step on a silicon wafer. The conducting lines are constructed by applying and structuring a gold layer on the mf-nitride. To selectively remove silicon, wet chemical etching is used. This fabrication step opens the membrane windows on which the conducting lines are carried.

\section{CPW modelling}

The model presented in (Heinrich, 1993) was applied to investigate the electromagnetic propagation properties of the CPWs. The model is based on the assumption of an infinitely expanded dielectric substrate beneath the coplanar metal lines. This assumption is well met for the case of conventional, several hundred $\mu \mathrm{m}$ thick substrates.

For the CPW fabricated in membrane technology, however, this assumption is not valid. Instead of a several hundred $\mu \mathrm{m}$ thick substrate we have a $t_{\mathrm{mem}}=1 \mu \mathrm{m}$ thick dielectric membrane over air. To calculate the effect of the membrane, we use an equivalent permittivity value $\varepsilon_{\mathrm{r}, \mathrm{LHP}}$ of a fictitious infinitely-expanded lower half-plane allowing to capture the dielectric influence of the thin membrane material on the equivalent lower half-plane in the model from (Heinrich, 1993).

To calculate $\varepsilon_{\mathrm{r}, \mathrm{LHP}}$, we first computed the capacitance per unit length $C_{\mathrm{mem}}^{\prime}$ of the membrane-based CPW with highprecision 2D-FEM simulations (QuickField ${ }^{\mathrm{TM}}, 5.5$ ). We then used a simple analytical relationship between $\varepsilon_{\mathrm{r}, \mathrm{LHP}}$ and $C_{\text {mem }}^{\prime}$, which can be derived from (Heinrich, 1993):

$\varepsilon_{\mathrm{r}, \mathrm{LHP}}=\frac{C_{\mathrm{mem}}^{\prime}}{2 \varepsilon_{0} F_{\text {low }}}-\frac{F_{\mathrm{up}}}{F_{\text {low }}}$

The expressions for $F_{\text {low }}$ and $F_{\text {up }}$ are given in (Heinrich, 1993), they depend only on the geometry of the CPW's cross section.
Table 1. Membrane CPW parameters and associated uncertainties.

\begin{tabular}{ccc}
\hline Parameter & value & half-width of pdf interval \\
\hline$w_{g}$ & $258 \mu \mathrm{m}$ & $0.25 \mu \mathrm{m}$ \\
$w$ & $166 \mu \mathrm{m}$ & $0.25 \mu \mathrm{m}$ \\
$s$ & $10 \mu \mathrm{m}$ & $0.25 \mu \mathrm{m}$ \\
$t$ & $1 \mu \mathrm{m}$ & $0.03 \mu \mathrm{m}$ \\
$\kappa$ & $27 \mathrm{MS} / \mathrm{m}$ & $1 \mathrm{MS} / \mathrm{m}$ \\
$\varepsilon_{\mathrm{r}, \mathrm{mem}}$ & 4.1 & 0.2 \\
$\varepsilon_{\mathrm{r}, \mathrm{LHP}}$ & 1.2707 & 0.0148 \\
$\tan \delta$ & 0.0001 & $5 \cdot 10^{-5}$ \\
\hline
\end{tabular}

Table 2. Virtual CPW parameters and associated uncertainties.

\begin{tabular}{ccc}
\hline Parameter & value & half-width of pdf interval \\
\hline$w_{g}$ & $258 \mu \mathrm{m}$ & $0.25 \mu \mathrm{m}$ \\
$w$ & $166 \mu \mathrm{m}$ & $0.25 \mu \mathrm{m}$ \\
$s$ & $10 \mu \mathrm{m}$ & $0.25 \mu \mathrm{m}$ \\
$t$ & $1 \mu \mathrm{m}$ & $0.03 \mu \mathrm{m}$ \\
$\kappa$ & $27 \mathrm{MS} / \mathrm{m}$ & $1 \mathrm{MS} / \mathrm{m}$ \\
$\varepsilon_{\mathrm{r}}$ & 4.1 & 0.2 \\
$\tan \delta$ & 0.0001 & $5 \cdot 10^{-5}$ \\
\hline
\end{tabular}

\section{CPW parameter sets}

The performance of three different CPWs against each other is compared in this paper. To calculate the broadband electrical properties of these CPWs, the model from (Heinrich, 1993) was used in the frequency range $1-110 \mathrm{GHz}$ in all three cases. The input quantities used in the CPW model are the width of the ground planes $w_{g}$, the center conductor width $w$, the width $s$ of the slot between center line and ground planes, the relative permittivity $\varepsilon_{\mathrm{r}, \mathrm{mem}}$, the dielectric loss tangent of the substrate $\tan \delta$, the thickness $t$ and the conductivity $\kappa$ of the metal layer, respectively (see Fig. 1). Tables 1-3 contain the parameters and associated uncertainties of the three CPWs under investigation. The assumptions regarding the propability density functions (pdf) will be discussed in the next section.

The parameters of the first CPW (see Table 1), constructed in membrane technology, are such that an impedance level near $50 \Omega$ is provided. $50 \Omega$ is the reference impedance of most instruments and connectors used in microwave scattering-parameter measurements. As a consequence of this requirement, the slot width is comparatively narrow with $s=10 \mu \mathrm{m}$. Otherwise, the impedance level would be much higher than in conventional thin-film technology on account of the absent substantial dielectric substrate. The dielectric constant $\varepsilon_{\mathrm{r}, \mathrm{LHP}}$ of the CPW in membrane technology is effective in the lower half plane of the model from (Heinrich, 1993). Using the equation specified in the previous section this effective dielectric constant was calculated to 
Table 3. $50 \Omega$-CPW parameters and associated uncertainties.

\begin{tabular}{ccc}
\hline Parameter & value & half-width of pdf interval \\
\hline$w_{g}$ & $280 \mu \mathrm{m}$ & $0.5 \mu \mathrm{m}$ \\
$w$ & $50 \mu \mathrm{m}$ & $0.5 \mu \mathrm{m}$ \\
$s$ & $23.5 \mu \mathrm{m}$ & $0.5 \mu \mathrm{m}$ \\
$t$ & $3.161 \mu \mathrm{m}$ & $0.25 \mu \mathrm{m}$ \\
$\kappa$ & $26.5 \mathrm{MS} / \mathrm{m}$ & $1 \mathrm{MS} / \mathrm{m}$ \\
$\varepsilon_{r}$ & 10.2 & 0.2 \\
$\tan \delta$ & 0.001 & $5 \cdot 10^{-5}$ \\
\hline
\end{tabular}

$\varepsilon_{\mathrm{r}, \mathrm{LHP}}=1.2707 \pm 0.0148$. This already points to one significant advantage of using membrane technology: the CPW is much less sensitive to uncertainties in the dielectric constant of the membrane.

The parameters of the second CPW (see Table 2) differ with regard to the first CPW in only one aspect: a dielectric bulk substrate replaces the dielectric membrane. The material parameters of this bulk substrate are identical to the supporting membrane $\left(\varepsilon_{\mathrm{r}}\right.$ in Table 2 equal to $\varepsilon_{\mathrm{r}, \mathrm{mem}}$ in Table 1). In order to apply the model from (Heinrich, 1993), the thickness of this bulk substrate is assumed to be large enough. This parameter set was chosen deliberately to illustrate the difference between the dielectric setup used in membrane and conventional thin-film technology, leaving the rest of the cross-sectional parameters unchanged. The parameter set of this CPW does not necessarily correspond to a CPW one would design in practice. Therefore, we called it a virtual CPW.

Finally, the parameters of the third CPW (see Table 3) were chosen from a realistic example of a $50 \Omega$-CPW fabricated on an alumina substrate. The dimensions of this CPW are close to the dimensions one can find on a commercial impedance standard substrate (ISS).

Offering the possibility to produce metallic structures with a very smooth surface and an almost perfect edge definition, an evaporation process for the metal lines of the first two CPWs is assumed. The metallic structures of the third CPW are produced by electroplating. This leads to higher fabrication deviations, but a more substantial metal thickness can be achieved.

Before investigating the uncertainties of the different CPW cases, we first examined the nominal electrical properties over a frequency range of $110 \mathrm{GHz}$. Fig. 2 presents the three data sets for the real and imaginary part of the propagation constant. The phase constant $\beta$ is normalized to its freespace value $\beta_{0}$. Apparently, the propagation characteristics of the membrane-supported CPW are better than for the other two CPWs discussed: the normalized phase constant is close to the ideal value of 1 , and the attenuation constant is lowest over frequency.
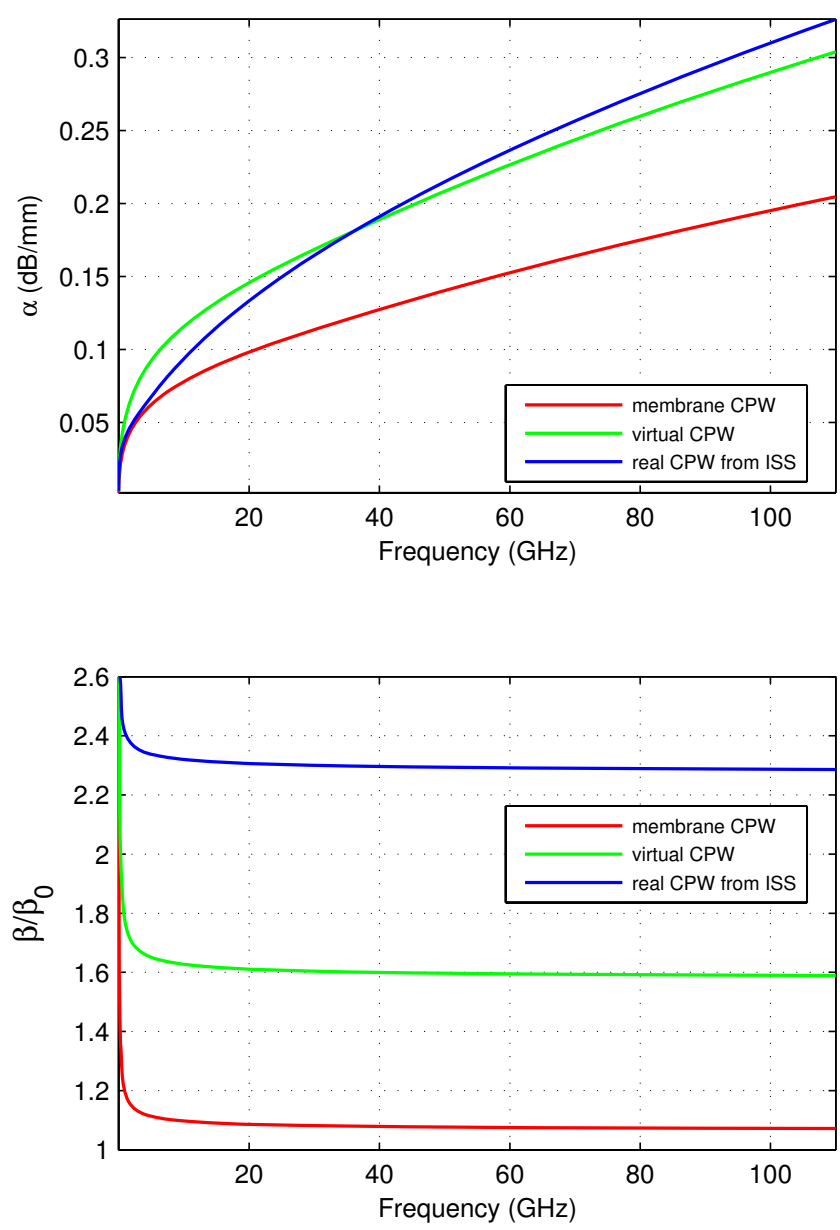

Fig. 2. Nominal values of propagation constant for all three CPW types discussed.

\section{Comparison of uncertainty distributions}

To study the propagation of uncertainties we use the Monte Carlo method as recommended in the Guide to the Expression of Uncertainty in Measurement (GUM), Supplement 1 (BIPM, 2008). The input quantities are assumed to be independent of each other as well as to have an uniform probability density function. Thus, the values of all input quantities are assumed to lie within an interval $[a, b]$ including a lower limit $a$ and an upper limit $b$. In Tables 1-3 the half-width of this interval $\frac{b-a}{2}$ is indicated for all input quantities.

As the output quantities of interest we investigated the real and imaginary part of the propagation constant as well as the magnitude and phase of the characteristic impedance at nine frequencies from 0.1 to $110 \mathrm{GHz}$. We first explored the composition of the uncertainty budgets over frequency in order to better understand the uncertainty mechanisms leading to the total observed uncertainty. 


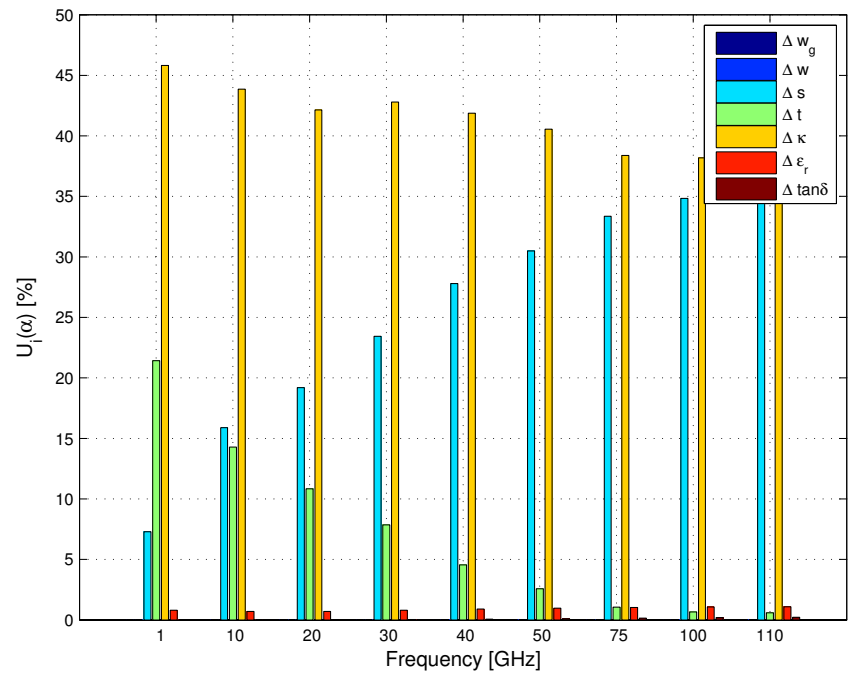

Fig. 3. Distribution of the uncertainties in the attenuation constant $\alpha$ for the membrane CPW (see Table 1).



Fig. 4. Distribution of the uncertainties in the attenuation constant $\alpha$ for the virtual CPW (see Table 2).

The percental contribution of each individual input quantity to the total standard deviation of the attenuation constant $\alpha$ is shown in Figs. 3-5. The total standard deviation was calculated using the adaptive Monte Carlo method of (BIPM, 2008) with statistics of the input quantities according to Tables $1-3$. Then the percental contribution was calculated as the ratio of the squared uncertainty contribution of the individual input quantity to the square of the total standard deviation. This ratio is sometimes termed uncertainty index $U_{i}$. In Figs. 3-5 each colored bar corresponds to the uncertainty index $U_{i}(\alpha)$, the contribution of a given input quantity (see legend) to the uncertainty in $\alpha$ at a given frequency.

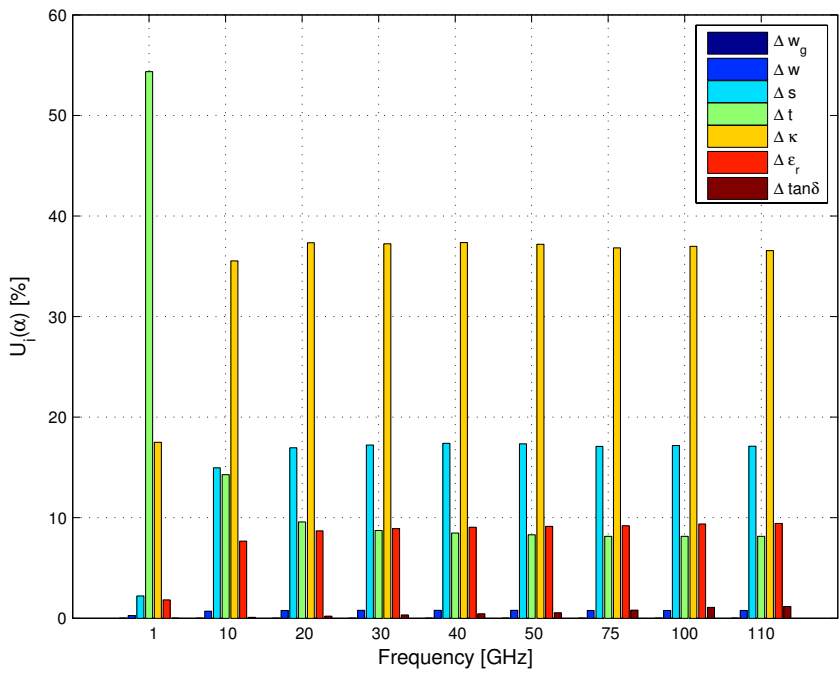

Fig. 5. Distribution of the uncertainties in the attenuation constant $\alpha$ for the $50-\Omega$ CPW (see Table 3 ).

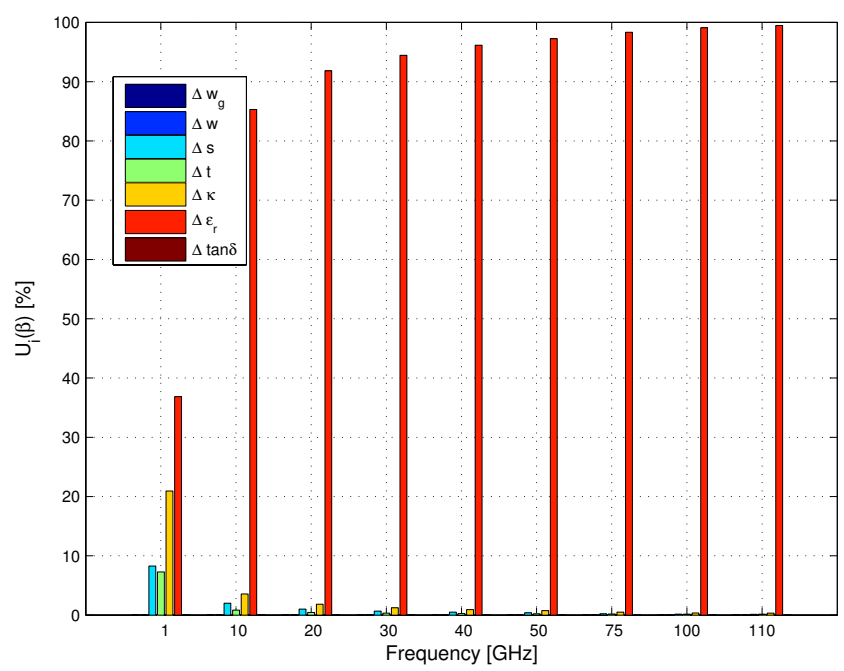

Fig. 6. Distribution of the uncertainties in the normalized phase constant $\beta$ for the membrane CPW (see Table 1).

For the virtual CPW and the $50 \Omega$-CPW (Figs. 4 and 5), at almost all frequencies the uncertainty in the bulk substrate's dielectric constant is an important contributor to the total uncertainty in $\alpha$. By contrast, the influence of the membrane's dielectric on the uncertainty in $\alpha$ can be neglected for the membrane supported CPW, illustrated in Fig. 3. The dominant influences in Fig. 3 stem from the thickness and the conductivity of the metal lines, with an increasing contribution of the gap definition at higher frequencies.

The cross-section's geometrical uncertainty is assumed to be rather low for the first and second CPW (within $\pm 0.25 \mu \mathrm{m}$ for $w_{g}, w$ and $s$ ). Figures 3 and 4 visualize the domination of the uncertainty budgets by the remaining input parameters. For the second CPW the uncertainties in the conductivity, the 


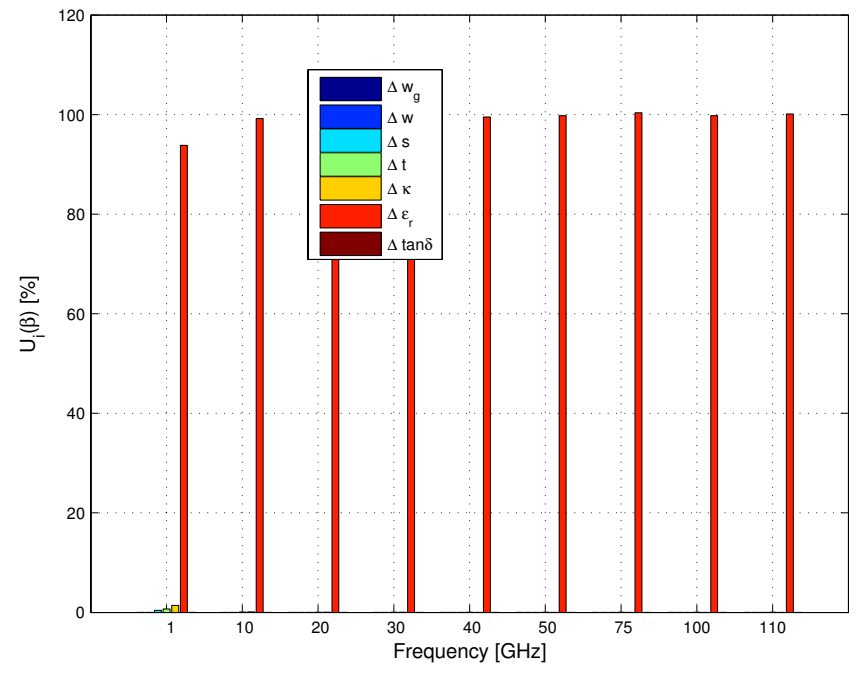

Fig. 7. Distribution of the uncertainties in the normalized phase constant $\beta$ for the virtual CPW (see Table 2).

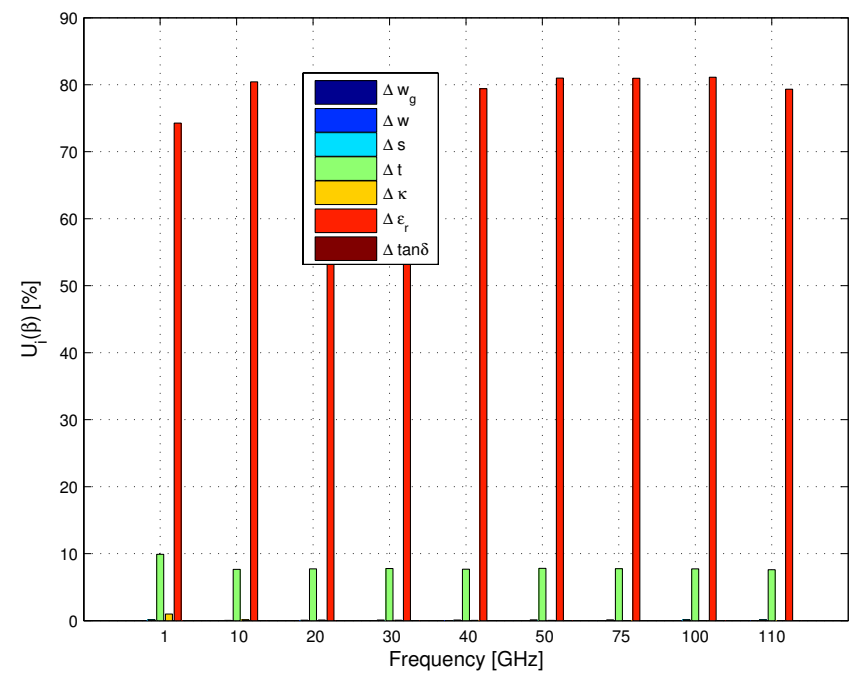

Fig. 8. Distribution of the uncertainties in the normalized phase constant $\beta$ for the 50- $\Omega$ CPW (see Table 3).

thickness and the permittivity play the biggest role according to Fig. 4. Despite the low level of uncertainty assumed, the uncertainty in the gap definition becomes more important for higher frequencies. For all three CPWs, the uncertainty in the conductivity is a dominant factor in the uncertainty budgets for the attenuation constant $\alpha$.

The uncertainty budgets over frequency for the phase constant normalized to the free-space value are represented in Figs. 6-8. The normalized phase constant is denoted with $\beta$ in this paper. We know from Arz et al., 2010 that the uncertainties in $\beta$ are dominated by the bulk substrate's dielectric properties for conventionally fabricated $\mathrm{CPW}$ and microstrip lines (MSL). Figure 6 demonstrates that this is also

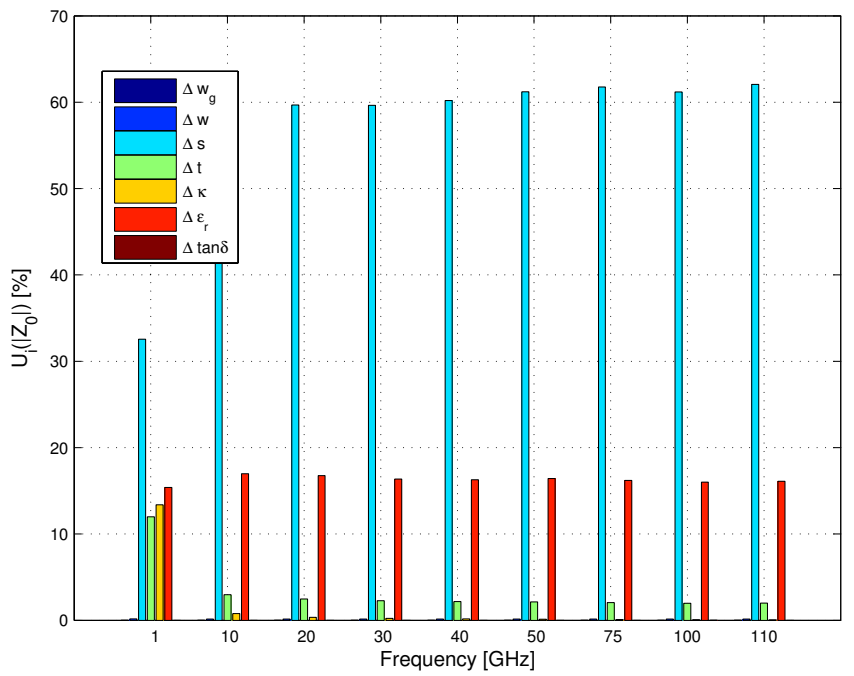

Fig. 9. Distribution of the uncertainties in the magnitude of the characteristic impedance $\left|Z_{0}\right|$ for the membrane CPW (see Table 1).

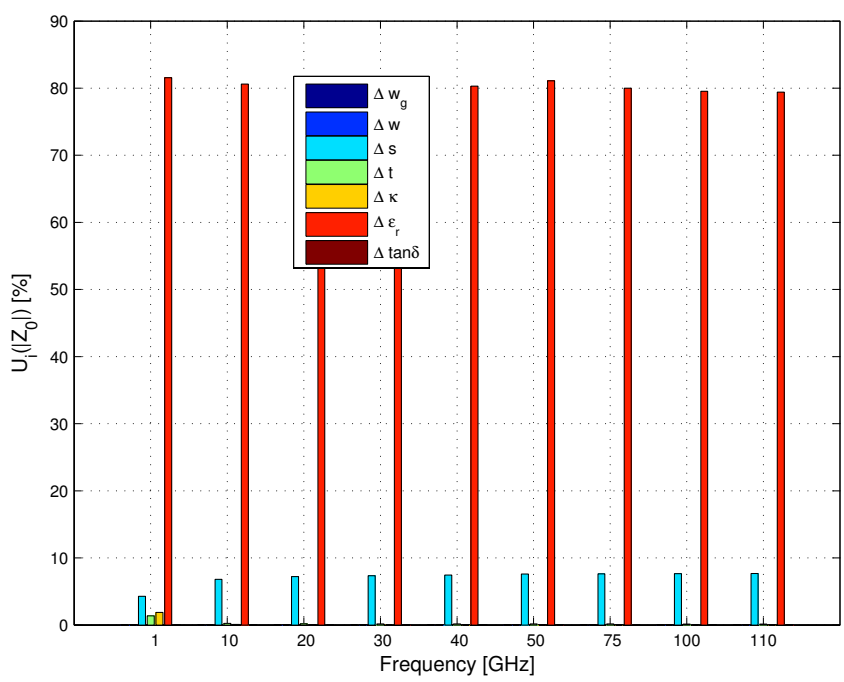

Fig. 10. Distribution of the uncertainties in the magnitude of the characteristic impedance $\left|Z_{0}\right|$ for the virtual CPW (see Table 2).

true of membrane-supported CPWs, even though the thickness of the membrane we assumed in our study was only $t_{\mathrm{mem}}=1 \mu \mathrm{m}$.

The uncertainty budgets over frequency for the magnitude of the characteristic impedance $Z_{0}$ are shown in Figs. 911. While the biggest contributor for the uncertainty budgets of the CPWs with a bulk substrate is the uncertainty in $\varepsilon_{\mathrm{r}}$ (Figs. 10-11), the uncertainty budget of the membranesupported CPW is dominated by the uncertainty in the width $s$ of the gap (Fig. 9).

Finally, Figs. 12-14 demonstrate the evolution over frequency of the uncertainty contributions to the phase of the characteristic impedance. For all three CPWs, the uncer- 


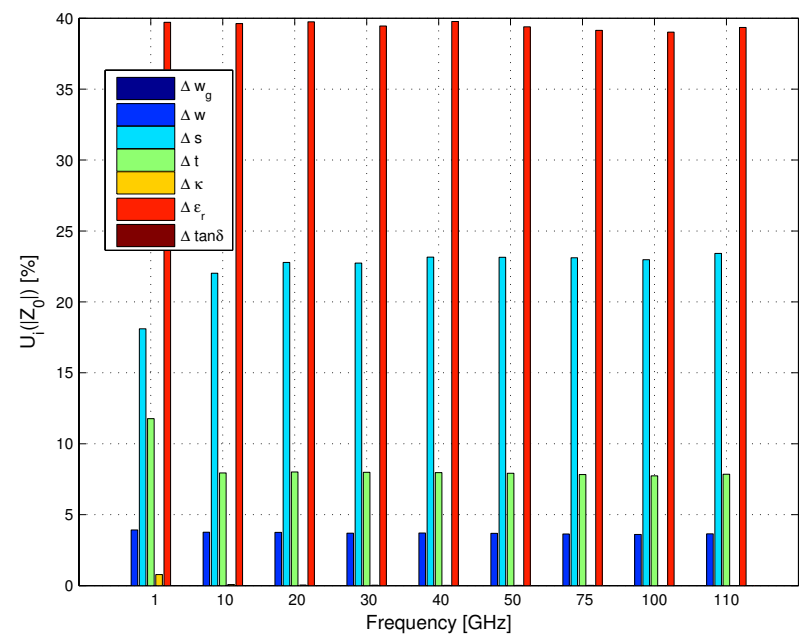

Fig. 11. Distribution of the uncertainties in the magnitude of the characteristic impedance $\left|Z_{0}\right|$ for the $50-\Omega$ CPW (see Table 3).

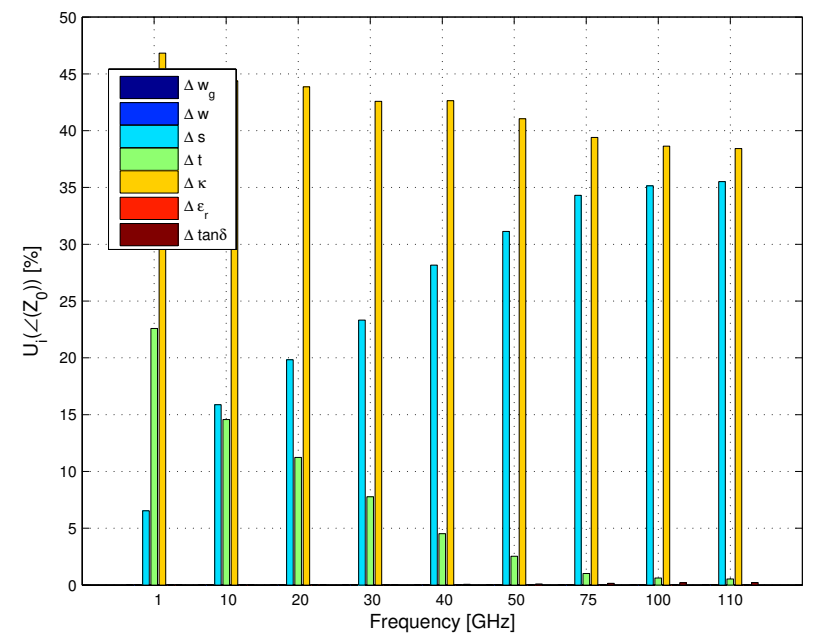

Fig. 12. Distribution of the uncertainties in the phase of the characteristic impedance for the membrane CPW (see Table 1).

tainty distributions look qualitatively quite similar. In all cases, the contributions of the uncertainty in the dielectric constant is negligible. From Figs. 12-14 one can conclude that, in terms of the uncertainty in $\angle\left(Z_{0}\right)$, the use of membrane technology does not offer any advantages.

\section{Comparison of total expanded uncertainties}

The absolute values of the uncertainties are not shown in Figs. 3-14. Therefore, we have summarized the total expanded uncertainties for all output quantities of the membrane-supported CPW in Table 4. We assumed a coverage factor of $k=2$. The ratio of the total uncertainties of either the first CPW to the second CPW (blue bars) or of the first CPW to the third CPW (brown bars) is plotted in

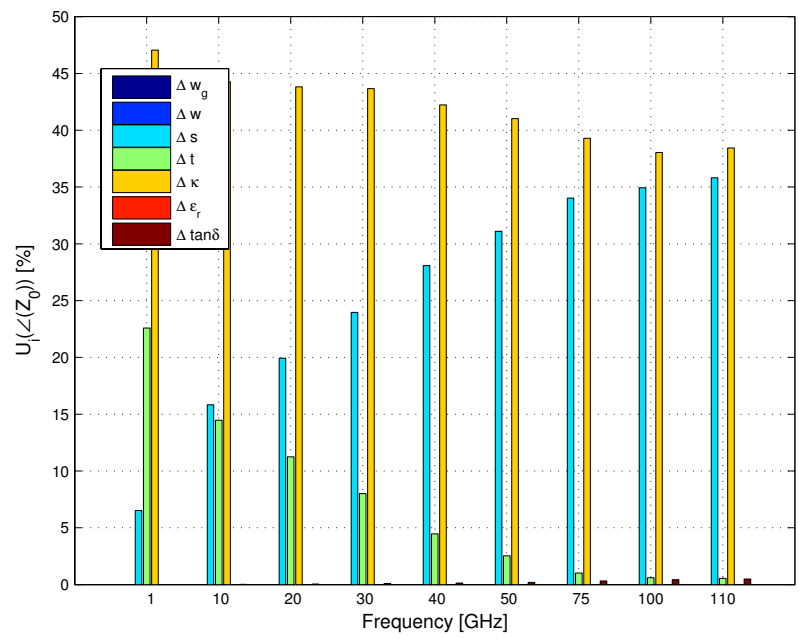

Fig. 13. Distribution of the uncertainties in the phase of the characteristic impedance for the virtual CPW (see Table 2).

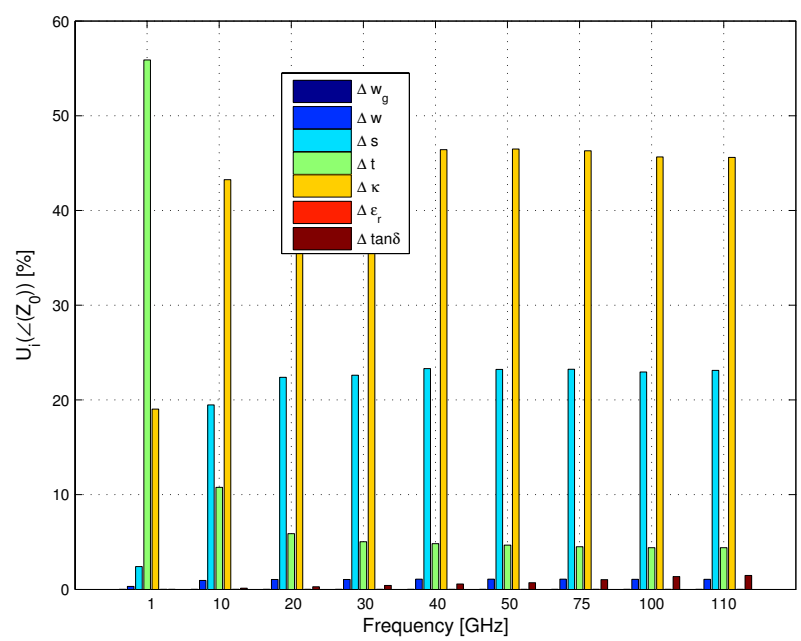

Fig. 14. Distribution of the uncertainties in the phase of the characteristic impedance for the $50-\Omega \mathrm{CPW}$ (see Table 3 ).

Figs. 15-18. This is equivalent to the reduction of the uncertainties when using membrane technology instead of conventional technology. The total uncertainties for the second and third CPW can be also derived from Table 4 together with Figs. 15-18.

The results for the uncertainties of the real and imaginary part of the propagation constant are illustrated in Figs. 1516. The uncertainty in $\alpha$ for the membrane-supported CPW is less than $70 \%$ of the corresponding uncertainty of either CPW with a bulk substrate for higher frequencies. The uncertainty in $\beta$ is reduced to values between 10 and $15 \%$. This is equivalent to an almost tenfold reduction of the uncertainty in $\beta$ by the use of membrane technology.

Figures 17-18 present the results for the uncertainties of the magnitude and phase of the characteristic impedance. For the magnitude of $Z_{0}$, the membrane technology reduces the 


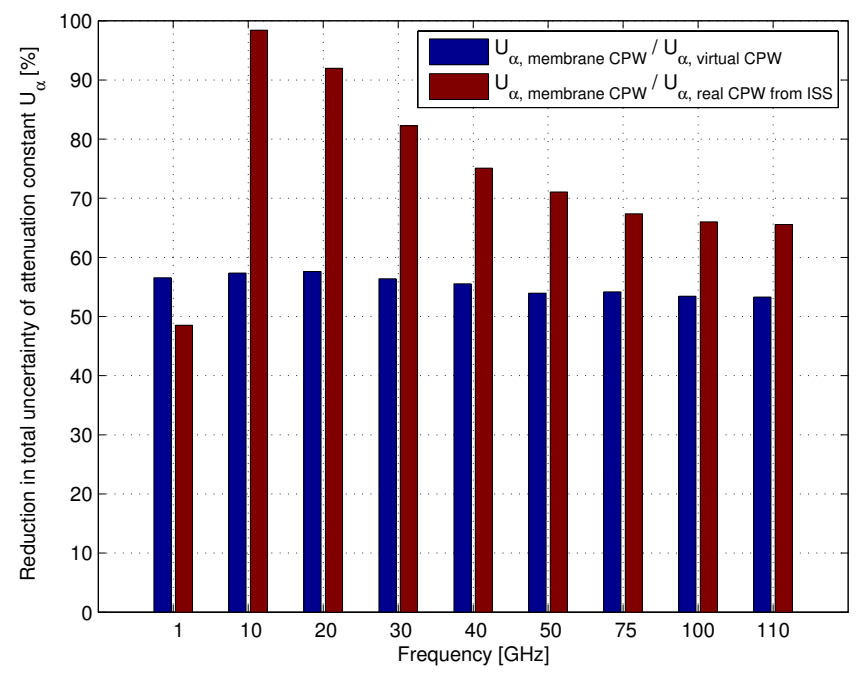

Fig. 15. Reduction of the uncertainties in $\alpha$ when using membrane instead of conventional technology.

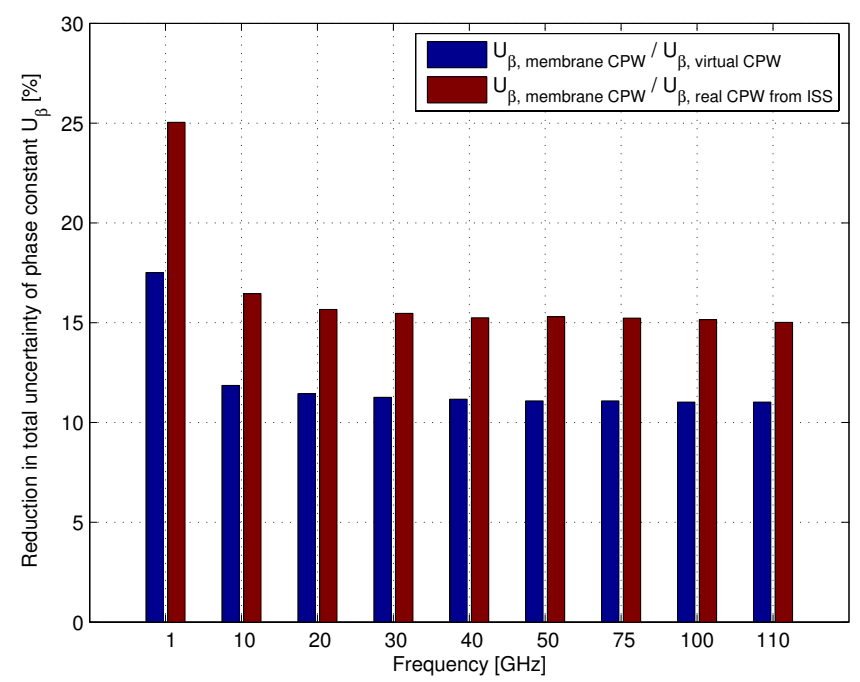

Fig. 16. Reduction of the uncertainties in $\beta$ when using membrane instead of conventional technology.

uncertainty to values between $\sim 55 \%$ (blue bars) and $\sim 75 \%$ (brown bars). No reduction is observed concerning the phase of $Z_{0}$. The comparison of the first and second CPW shows identical values, while the total uncertainty in $L\left(Z_{0}\right)$ for the $50-\Omega \mathrm{CPW}$ is even lower than for the membrane-supported CPW. This is consistent with the conclusions drawn from Figs. 12-14: the uncertainty in the phase of $Z_{0}$ does not depend on the uncertainty in $\varepsilon_{\mathrm{r}}$.

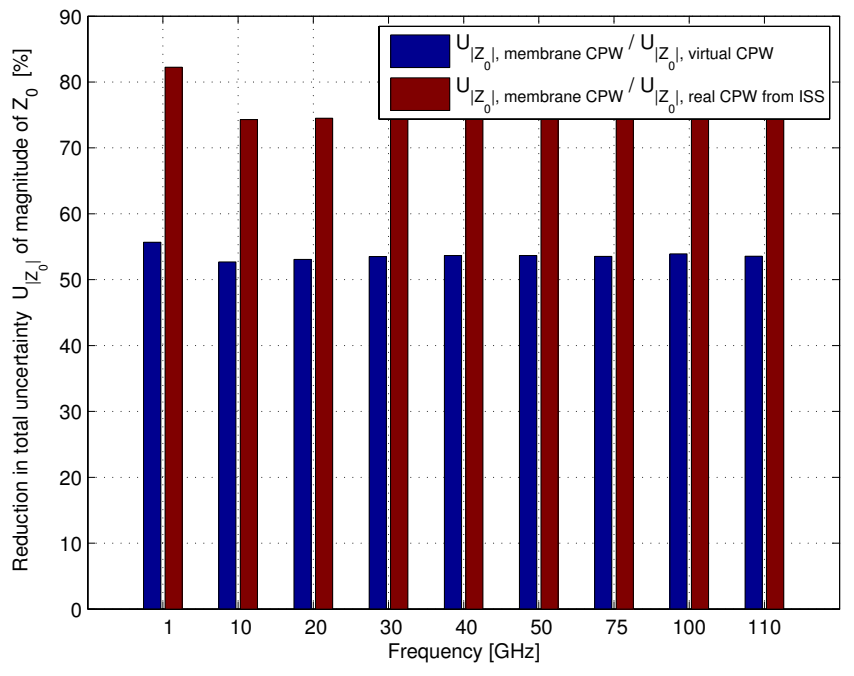

Fig. 17. Reduction of the uncertainties in $\left|Z_{0}\right|$ when using membrane instead of conventional technology.

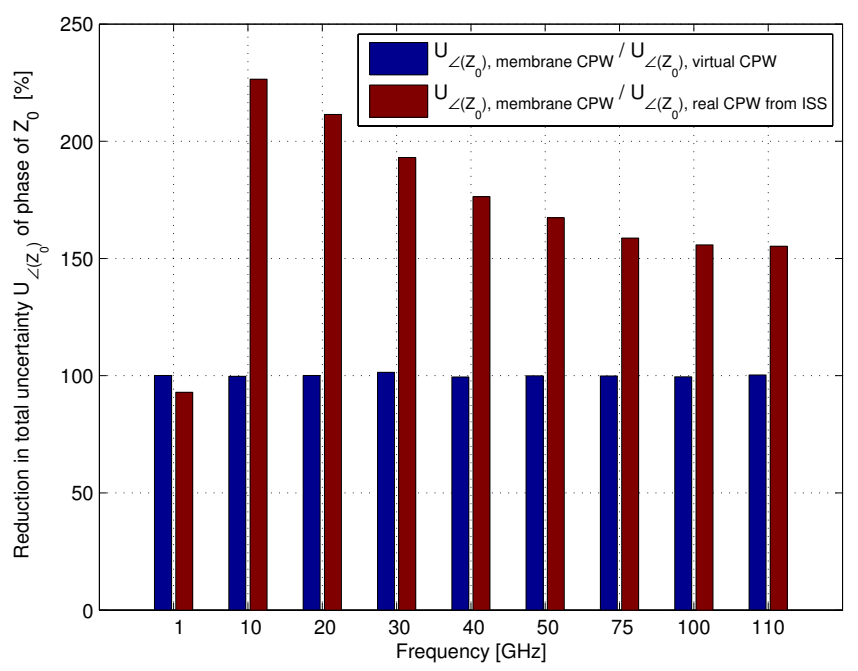

Fig. 18. Reduction of the uncertainties in phase of $Z_{0}$ when using membrane instead of conventional technology.

\section{Conclusions}

In this work, we presented a closer examination of the uncertainties in the electrical properties of a CPW fabricated in membrane technology compared to CPWs built on several hundred $\mu \mathrm{m}$ thick dielectric substrates. An expression was derived for the dielectric constant of an equivalent half" space beneath the signal conductors. This was used to describe the dielectric effect of the supporting membrane, in order to be able to use a broadband analytic CPW model for the membrane-supported CPW.

The sensitivities of the propagation constant and the characteristic impedance were analyzed while allowing for tolerances in the cross-sectional CPW parameters for frequencies 
Table 4. Total expanded uncertainties for membrane-supported CPW.

\begin{tabular}{ccccc}
\hline $\mathrm{f}[\mathrm{GHz}]$ & $U_{\alpha}[\mathrm{dB} / \mathrm{mm}]$ & $U_{\beta}$ & $U_{\left|Z_{0}\right|}[\Omega]$ & $U_{L_{Z_{0}}}[\mathrm{rad}]$ \\
\hline 1 & 0.0012 & 0.0058 & 0.55 & 0.0048 \\
10 & 0.0028 & 0.0035 & 0.48 & 0.0013 \\
20 & 0.0035 & 0.0034 & 0.47 & 0.0008 \\
30 & 0.0038 & 0.0033 & 0.48 & 0.0006 \\
40 & 0.0040 & 0.0033 & 0.48 & 0.0005 \\
50 & 0.0042 & 0.0032 & 0.47 & 0.0004 \\
75 & 0.0050 & 0.0032 & 0.48 & 0.0003 \\
100 & 0.0056 & 0.0032 & 0.48 & 0.0003 \\
110 & 0.0059 & 0.0032 & 0.48 & 0.0003 \\
\hline
\end{tabular}

from 1 to $110 \mathrm{GHz}$. We were able to demonstrate that, for the CPW parameter sets chosen in this study, an almost tenfold reduction in the uncertainty of the phase constant $\beta$ can be achieved through the use of membrane technology. Moderate improvements of the uncertainty can be obtained for the attenuation constant and the magnitude of the characteristic impedance. Membrane technology does not offer any improvements for output quantities that do not depend on the dielectric constant of the substrate, like e.g. the phase of the characteristic impedance.

Acknowledgements. The authors would like to thank the Test and Measurement Division, Rohde \& Schwarz GmbH \& Co. KG, München, Germany for providing the membrane material.

\section{References}

Arz, U., Leinhos, J., and Janezic, M. D.: Effect of Material Properties on Broadband Electrical Behavior of Coplanar Waveguides, Conference on Precision Electromagnetics CPEM, Broomfield, Colorado, 8-13 June 2008, 470-471, doi:10.1109/CPEM.2008.4574857, 2008a.

Arz, U., Leinhos, J., and Janezic, M. D.: Broadband Dielectric Material Characterization: A Comparison of On-Wafer and SplitCylinder Measurements, 38th European Microwave Conference EuMC, 27-31 October 2008 , Amsterdam, Netherlands, 913916, doi:10.1109/EUMC.2008.4751602, 2008b.

Arz, U. and Kuhlmann, K.: Uncertainties in Coplanar Waveguide and Microstrip Line Standards for On-Wafer ThruReflect-Line Calibrations, 75th ARFTG Microwave Measurement Conference, 28-28 May 2010, Anaheim, California, 1-5, doi:10.1109/ARFTG.2010.5496328, 2010.

BIPM, IEC, IFCC, ISO, IUPAC, IUPAP, and OIML: Evaluation of measurement data - Supplement 1 to the "Guide to the expression of uncertainty in measurement": Propagation of distributions using a Monte Carlo method, 1, BIPM Joint Committee for Guides in Metrology, JCGM 101:2008, Paris, 2008.

Dib, N., Harokopus Jr., W. P., Katehi, L. P., Ling, C. C., and Rebeiz, G. M.: Study of a Novel Planar Transmission Line, IEEE MTT-S, 10-14 June 1991, Boston, 2, 623-626, doi:10.1109/MWSYM.1991.147080, 1991.

Heinrich, W.: Quasi-TEM description of MMIC coplanar lines including conductor-loss effects, IEEE T MICROW THEORY, 41, 45-52, doi:10.1109/22.210228, 1993.

Katehi, L. P. and Rebeiz, G. M.: Novel micromachined approaches to MMICs using low-parasitic, high-performance transmission media and environments, IEEE MTT-S, 1721 June 1996, San Francisco, California, 2, 1145-1148, doi:10.1109/MWSYM.1996.511232, 1996.

QuickField ${ }^{\mathrm{TM}} 5.5$, Tera Analysis Ltd..

Weller, T. M., Katehi, L. P., Herman, M. I., and Wamhof, P. D.: Membrane technology (MIST-T) applied to microstrip: a $33 \mathrm{GHz}$ Wilkinson power divider, IEEE MTTS, 23-27 May 1994, San Diego, California, 2, 911-914, doi:10.1109/MWSYM.1994.335209, 1994. 